\title{
Seeing Scenes: Topographic Visual Hallucinations Evoked by Direct Electrical Stimulation of the Parahippocampal Place Area
}

\author{
Pierre Mégevand, ${ }^{1}$ David M. Groppe, ${ }^{1}$ Matthew S. Goldfinger, ${ }^{1}$ Sean T. Hwang, ${ }^{2}$ Peter B. Kingsley, ${ }^{3}$ Ido Davidesco, ${ }^{4}$ \\ and Ashesh D. Mehta ${ }^{1}$ \\ Departments of ${ }^{1}$ Neurosurgery, Feinstein Institute for Medical Research, ${ }^{2}$ Neurology, and ${ }^{3}$ Radiology, Hofstra North Shore-LIJ School of Medicine, \\ Manhasset, New York 11030, and ${ }^{4}$ Neuroscience Institute, Princeton University, Princeton, New Jersey 08540
}

In recent years, functional neuroimaging has disclosed a network of cortical areas in the basal temporal lobe that selectively respond to visual scenes, including the parahippocampal place area (PPA). Beyond the observation that lesions involving the PPA cause topographic disorientation, there is little causal evidence linking neural activity in that area to the perception of places. Here, we combined functional magnetic resonance imaging (fMRI) and intracranial EEG (iEEG) recordings to delineate place-selective cortex in a patient implanted with stereo-EEG electrodes for presurgical evaluation of drug-resistant epilepsy. Bipolar direct electrical stimulation of a cortical area in the collateral sulcus and medial fusiform gyrus, which was place-selective according to both fMRI and iEEG, induced a topographic visual hallucination: the patient described seeing indoor and outdoor scenes that included views of the neighborhood he lives in. By contrast, stimulating the more lateral aspect of the basal temporal lobe caused distortion of the patient's perception of faces, as recently reported (Parvizi et al., 2012). Our results support the causal role of the PPA in the perception of visual scenes, demonstrate that electrical stimulation of higher order visual areas can induce complex hallucinations, and also reaffirm direct electrical brain stimulation as a tool to assess the function of the human cerebral cortex.

Key words: direct electrical stimulation; functional magnetic resonance imaging; intracranial electroencephalography; parahippocampal place area; scene perception; visual hallucination

\section{Introduction}

A sense of place is paramount to our ability to recognize and navigate the world around us. During the last century, several observations of patients with topographic disorientation have implicated brain areas at the junction of the basal temporal and occipital lobes, predominantly in the right hemisphere, in this ability (Landis et al., 1986). More recently, functional neuroimaging has disclosed a network of cortical areas that selectively activate when viewing scenes or buildings, including the parahippocampal place area (PPA; Aguirre et al., 1998; Epstein and Kanwisher, 1998). The PPA responds to visual scenes, whether familiar or not, in a viewpoint-specific man-

\footnotetext{
Received Dec. 12, 2013; revised Feb. 10, 2014; accepted March 4, 2014.

Author contributions: P.M., D.M.G., and A.D.M. designed research;P.M., D.M.G., M.S.G., S.T.H., P.B.K., and A.D.M. performed research; P.M., D.M.G., I.D., and A.D.M. analyzed data; P.M., D.M.G., S.T.H., I.D., and A.D.M. wrote the paper.

This work was supported by the Page and Otto Marx Jr Foundation, and Swiss National Science Foundation (Grant P3SMP3_148388 to P.M.). We thank the patient for his participation and Willie Walker Jr, the physicians, and other professionals of the Neurosurgery and Neurology departments of North Shore University Hospital for their assistance.

The authors declare no competing financial interests.

Correspondence should be addressed to Dr Ashesh Mehta, Department of Neurosurgery, Hofstra North Shore-LIJ School of Medicine and Feinstein Institute for Medical Research, 300 Community Drive, Manhasset, NY 11030. E-mail:amehta@nshs.edu.

DOI:10.1523/JNEUROSCI.5202-13.2014

Copyright $\odot 2014$ the authors $\quad 0270-6474 / 14 / 345399-07 \$ 15.00 / 0$
}

ner (Epstein, 2008), and with low response latencies, as evidenced by recent intracranial EEG (iEEG) work (Bastin et al., 2013a,b). Patients who sustain lesions involving this area have trouble navigating both familiar and unfamiliar environments (Epstein et al., 2001). These results suggest that the PPA is necessary for the representation of local visual scenes (Epstein, 2008).

Apart from the aforementioned lesion studies, there is little causal evidence linking neural activity in the PPA to the visual perception of scenes. Penfield's pioneering work demonstrated complex visual perceptions elicited by electrical stimulation of the temporal and frontal lobes (Penfield and Perot, 1963; Blanke et al., 2000). However, topographic hallucinations have not been reported upon stimulation of scene-selective visual cortex. In one cortical stimulation study where visual areas were identified by fMRI, stimulation of the PPA did not elicit any complex visual hallucination (Murphey et al., 2009).

Here, we report the results of a multimodal investigation into the function of place-selective cortex in the basal temporal lobe in a patient implanted with intracranial electrodes as part of the surgical approach to drug-resistant focal epilepsy. A placeselective area in the posterior collateral sulcus and medial fusiform gyrus was identified using functional MRI (fMRI) and iEEG. Direct electrical stimulation of that area elicited topographic visual hallucinations. 


\section{Patients and Methods}

Patient and recordings. A 22-year-old left-handed male was referred for surgical evaluation for medically refractory epilepsy, which appeared after West Nile viral encephalitis at the age of 10 . He had no other epilepsy risk factors, with normal developmental milestones before the onset of his epilepsy. Diurnal seizures were preceded by déjà vu, described by the patient as a sense of familiarity with his circumstances or situation. He also reported weightlessness and otherworldliness. He denied the actual environment appearing abnormal or unrecognizable, and did not report any visual hallucinations or obscuration. Seizures would then typically proceed to alteration of consciousness, with rare secondary generalization.

The neurological examination was unrevealing. Neuropsychological testing showed normal verbal and visual memory, but impaired spatial memory. Specifically, in a test where faces were presented on a grid, the patient's performance on remembering the position of the faces after a delay was far below expected levels, whereas his ability to identify the previously shown faces among distractors was low normal. Magnetic resonance imaging demonstrated bilateral hippocampal sclerosis and atrophy. Scalp video-EEG monitoring revealed right temporal interictal sharp-wave discharges and slowing, and a clinically subtle right temporal seizure. Positron emission tomography of the brain was concordant with right greater than left infero-basal temporal hypometabolism. Wada testing showed bilateral language representation and relatively preserved memory function on both sides.

The patient was stereotactically implanted with 10 depth electrode shafts bearing a total of 88 contacts ( $5 \mathrm{~mm}$ intercontact spacing; Ad-Tech Medical Instrument) into the right frontal and temporal lobes. The patient provided informed consent under the guidelines of the local institutional review board.

MRI acquisition and analysis. Before electrode implantation, anatomical and functional MRI data were acquired on a 3-tesla Signa HDx scanner (GE Healthcare). The anatomical scan consisted of an isometric, $1 \mathrm{~mm} 3 \mathrm{D}$ T1-weighted sequence. During the fMRI scan, the patient performed a 1-back memory task in a standard visual category localizer experiment (Davidesco et al., 2013). Grayscale pictures of faces, buildings, man-made objects, and geometric patterns (Fig. $1 C$ ) were presented in blocks of $10 \mathrm{~s}$, followed by a $6 \mathrm{~s}$ blank screen. Each image was presented for $250 \mathrm{~ms}$, followed by a $750 \mathrm{~ms}$ blank screen. Blood oxygen leveldependent contrast images were obtained with gradient-echo echoplanar imaging sequence (field-of-view $220 \mathrm{~mm}, 3.5 \times 3.5 \times 4 \mathrm{~mm}$ voxel size, $64 \times 64$ matrix, flip angle $70^{\circ}$, repetition time $2 \mathrm{~s}$, echo time $30 \mathrm{~ms}$, axial acquisition plane, 210 contiguous volumes).

fMRI data were analyzed with the FSL software package (Jenkinson et al., 2012). The first three volumes were discarded. Preprocessing included 3D motion correction, linear trend removal, filtering out low frequencies ( $32 \mathrm{~s}$ high pass filter cutoff), and $5 \mathrm{~mm}$ spatial smoothing. A general linear model analysis was conducted to identify category-specific activation. Place-related regions were identified using the contrast of houses versus all other categories and face-related regions using the contrast of faces vs. all other categories (fixed effects analysis). A clusterbased permutation test was used to correct for multiple comparisons using a cluster inclusion $z$-score threshold of 2.6 and a family-wise error rate of 0.01 . Functional images were superimposed on $2 \mathrm{D}$ anatomical images through a rigid transformation and trilinear interpolation.

Anatomical localization of electrodes. After implantation, electrode locations were visually identified on a thin-slice brain CT (Somatom Definition AS, Siemens) using BioImage Suite (Papademetris et al., 2011). To aid coregistration of the CT scan to the preimplant MRI, a postimplant T1-weighted anatomical MRI was acquired on a 1.5-tesla Signa Excite scanner (GE Healthcare) as well. The electrode locations were then mapped to the preimplant MRI via a rigid transformation derived from coregistering the preimplant and postimplant MRIs and postimplant MRI and CT scans using FLIRT (Jenkinson and Smith, 2001) and the skull-stripping BET algorithm (Smith, 2002), both part of FSL.

Intracranial EEG acquisition and analysis. Intracranial EEG signals were referenced to a subdermal vertex electrode, filtered (analog bandpass, with half-power boundaries at 0.1 and $200 \mathrm{~Hz}$ ), digitized at $500 \mathrm{~Hz}$ and stored for offline analysis by an XLTEK EMU128FS system (Natus Medical). While iEEG was recorded, the patient viewed a series of 200 grayscale images, $\sim 15^{\circ} \times 15^{\circ}$ visual angle in size, presented for $250 \mathrm{~ms}$ once every second and performed a 1-back memory task. Images belonged to one of six categories: houses $(n=10)$, faces (14), cartoon faces (5), body parts (5), tools (10), and abstract patterns (5; Fig. 1C). Each image was presented four times, except for two body-part images that were presented five and seven times respectively. Images in each category could serve as targets $(n=25)$. The patient was instructed to focus his eyes on a fixation cross at the center of the display and a small white fixation dot was superimposed on each stimulus to help maintain fixation (Davidesco et al., 2013). The task was implemented using Presentation (Neurobehavioral Systems) and a laptop.

iEEG responses to targets were excluded from analysis to avoid confounds from stimulus repetition and the detection of rare target trials. iEEG responses to cartoon faces were also ignored. Moreover, one trial was excluded due to a large interictal spike, which left 35 house, 49 face, and 73 body/tool/pattern trials in the analysis. To assess the local neural response to each stimulus, we measured changes in broadband gamma/ high-gamma power (BBGP) between $40-160 \mathrm{~Hz}$, as was done in a comparable study by Parvizi et al. (2012). Specifically, bipolar iEEG derivations were obtained from pairs of neighboring electrodes to make the data as comparable as possible to bipolar electrical stimulation. BBGP was then estimated via a $100 \mathrm{~ms}$ moving time window ( $2 \mathrm{~ms}$ steps) and a discrete Fourier transform (DFT) using two Slepian tapers as implemented by the Chronux MATLAB toolbox (Bokil et al., 2010). This provides a frequency resolution of $10 \mathrm{~Hz}$ and a frequency resolution bandwidth of $30 \mathrm{~Hz}$. Before the DFT, the mean of each $100 \mathrm{~ms}$ window was removed to dampen low-frequency activity. Spectral power estimates for each trial were transformed into decibels and averaged from 40 to $160 \mathrm{~Hz}$. BBGP in a prestimulus baseline $(-150$ to $0 \mathrm{~ms})$ for each trial was then removed from the BBGP values across the entire trial to heighten stimulus-related changes in BBGP. We tested for selectively enhanced BBGP responses to houses or faces versus other stimuli using a one-tailed independent-samples $t$ test at each electrode pair and time point from 50 to $750 \mathrm{~ms}$. The Benjamini-Hochberg false discovery rate control algorithm (Benjamini and Hochberg, 1995) was used to adjust the $p$ values for each family of $t$ tests and thus limit the expected proportion of false-positives to $1 \%$ (Groppe et al., 2011). To maximize statistical power, analyses were limited to the two electrode shafts in the basal temporal lobe (DTIp and DHb), where face- and place-selective visual areas were previously described (Parvizi et al., 2012; Bastin et al., 2013b).

Electrical brain stimulation. Bipolar electrical stimulation was delivered to neighboring contacts along the shaft of a stereotaxic electrode using 200- $\mu$ s-long, square-wave biphasic pulses at $50 \mathrm{~Hz}$ for $\sim 2 \mathrm{~s}$ (S12 stimulator, Grass Technologies). The intensity was ramped up in $1 \mathrm{~mA}$ increments from 1 to $4 \mathrm{~mA}$ or until afterdischarges were observed, in which case the next stimulation was held off until the afterdischarges subsided. Before stimulating each site, the patient was asked to report if he saw, heard, or felt anything. He was asked to keep his eyes open during the procedure and did not perform any task. Sham stimulation (asking the patient to report his percepts without injecting any current into the electrodes) was not performed. Table 1 lists stimulation sites, durations, afterdischarges, and transcripts of the patient's reports. Although every contact was stimulated at least once, the current report focuses on the responses from electrode shafts DTIp (20 stimulation trials at 7 sites) and DHb ( 7 trials at 4 sites). Before DTIp was stimulated, 23 stimulation trials were performed at nine sites in the superior temporal lobe; between DTIp and $\mathrm{DHb}, 74$ stimulations were delivered at 29 sites in the anterior temporal lobe and in the frontal lobe. Twenty-seven minutes separated stimulations at DTIp and DHb.

\section{Results}

\section{Localization of house-selective visual responses by fMRI and iEEG}

fMRI revealed selective activation to houses bilaterally in the posterior basal temporal lobe, most prominently in the collateral sulcus and medial fusiform gyrus (Fig. 1B). Electrodes DTIp5 to 

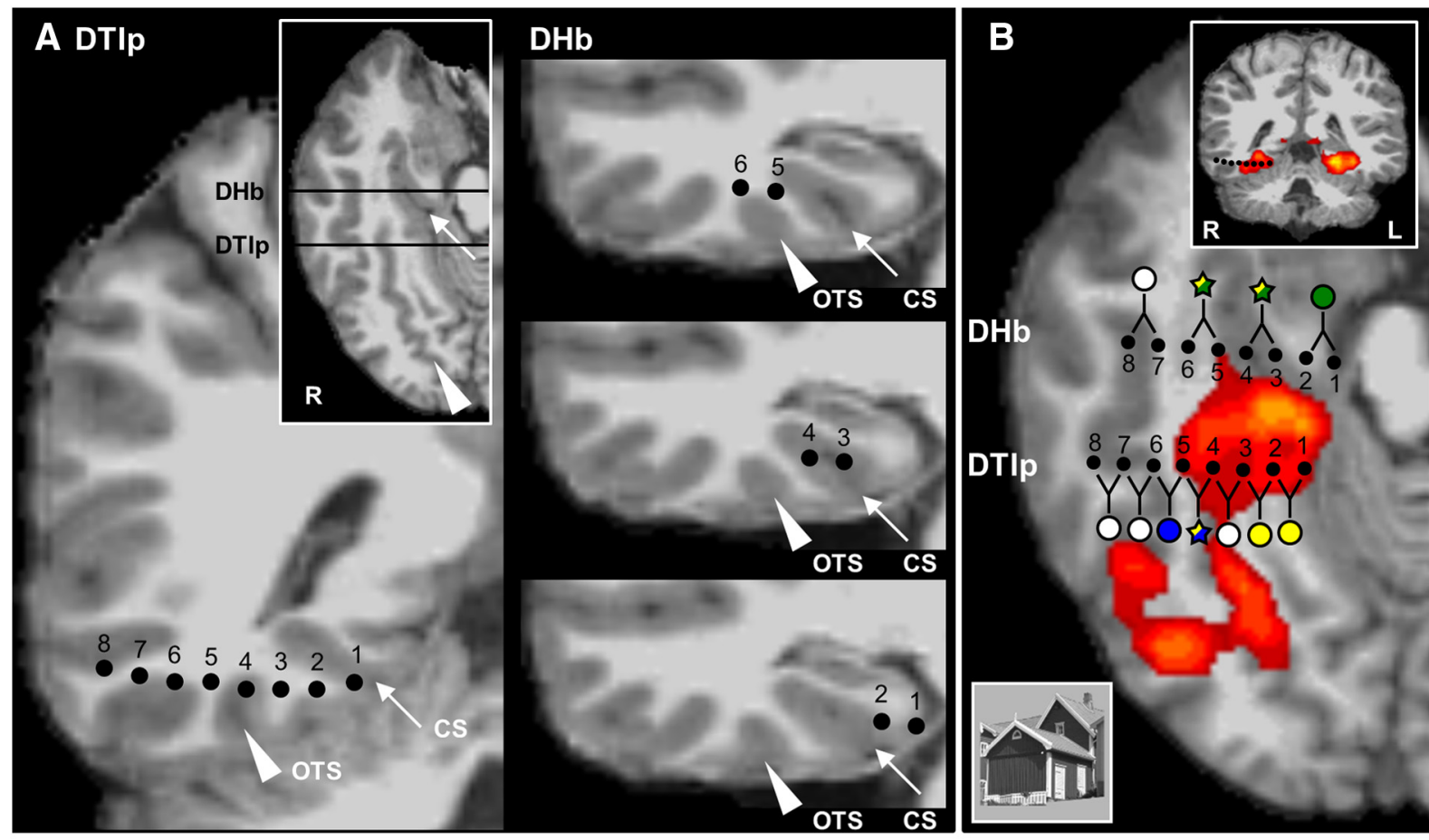

C

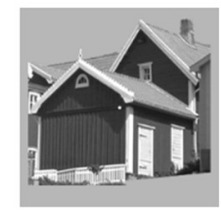

house

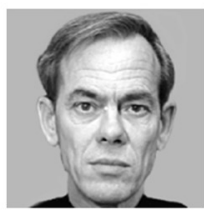

face

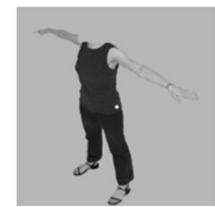

body

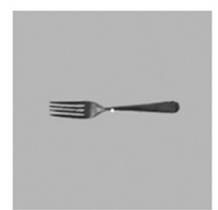

tool

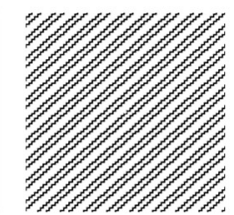

pattern
O No percept
O Topographical hallucination
Face distortion
Déjà vu
As Afterdischarge
D

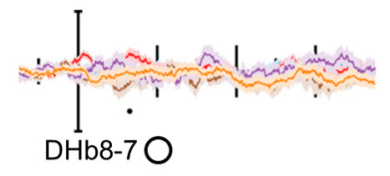

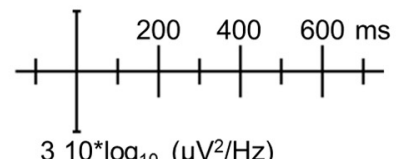

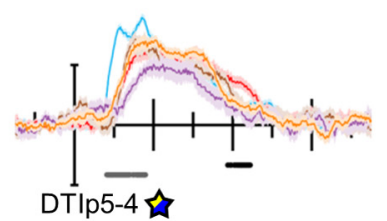

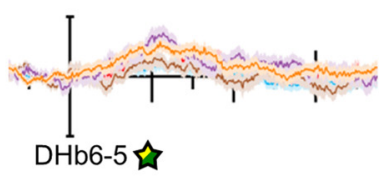

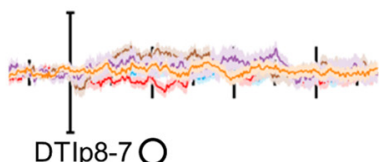

DTIp8-7 O

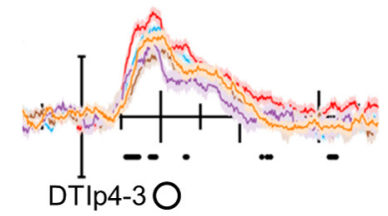

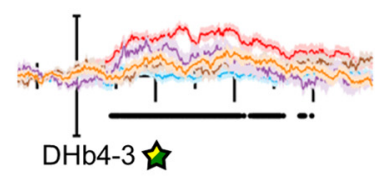<smiles>CC(C)(C)[13C](=O)O</smiles>

DTIp7-6 O

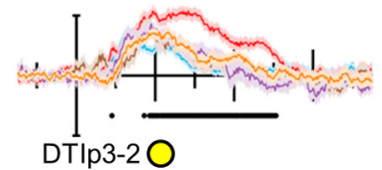

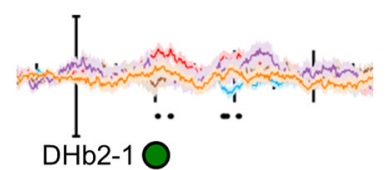

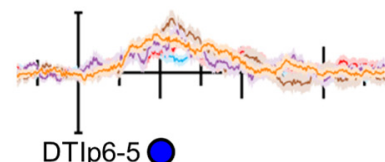

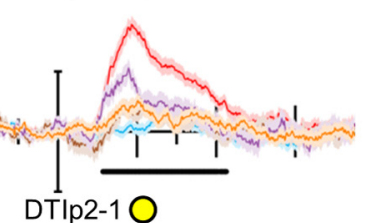

Figure 1. Location of the intracranial electrodes, house-specific visual responses identified by fMRI, iEEG, and results of electrical brain stimulation. $\boldsymbol{A}$, The location of the intracranial electrodes of shafts DTIp and DHb is displayed on the patient's high-resolution T1 MRI. Inset, Illustration of the coronal slices along the rostral-caudal axis. All DTIp electrodes were in the same coronal plane. For DHb, contacts 4 and 3 were on the coronal slice located in the inset; contacts 6 and 5 were on the coronal slice immediately rostral; and contacts 2 and 1 on the slice immediately caudal. The white arrow highlights the collateral sulcus; white arrowhead, the occipitotemporal sulcus. $\boldsymbol{B}$, Regions that displayed greater B0LD responses to houses than other visual stimuli are overlaid on the patient's isometric axial T1 MRI. The basal temporal electrodes DHb and DTIp are also displayed. The inset shows a coronal slice in the plane of DTIp. The effect of direct electrical stimulation of pairs of neighboring electrodes is depicted as colored circles, or stars if there were afterdischarges as a result of the stimulation. The color codes for the symbols are detailed in C. The axial slice shown was in the plane of contacts DTIp 5 through 2 and of contacts DHb4 and 3 . DTIp7 and 1 and DHb6 and 5 were on the slice immediately $(1 \mathrm{~mm}$ ) dorsal and were projected down; DTIp8 was $2 \mathrm{~mm}$ dorsal and was also projected down; DHb2 and 1 were $1 \mathrm{~mm}$ ventral and were projected up. C, Left, Exemplars of stimuli used in iEEG paradigm to detect visual category-selective cortex. The fMRI paradigm included houses, faces, tools and patterns but not body parts. Right, Color codes for the symbols used to summarize the results of electrical stimulation in $\boldsymbol{B}$ and $\boldsymbol{D}$ (see Results; Table 1). $\boldsymbol{D}$, Event-related changes in iEEG broadband gamma/high gamma power ( $40-160 \mathrm{~Hz}$ ) following presentation of five categories of images. Different categories are represented by different colors (C). Shading indicates SE. Black dotted lines below each plot indicate significantly $\left(p_{\text {adj }}<0.01\right)$ higher responses to places than other visual stimuli. Gray lines indicate significantly $\left(p_{\text {adj }}<0.01\right)$ higher responses to faces than other visual stimuli. The symbols next to the electrode names summarize the effects of direct electrical stimulation at each site. 
Table 1. Details of the electrical brain stimulation procedure

\begin{tabular}{|c|c|c|c|c|}
\hline Site & $\begin{array}{l}\text { Amplitude } \\
(\mathrm{mA})\end{array}$ & $\begin{array}{l}\text { Duration } \\
\text { (s) }\end{array}$ & ADs? & Unedited transcripts of the patient's and experimenters' dialogue \\
\hline \multirow[t]{5}{*}{ DTlp4-3 } & 4 & 2 & DTlp5-1 & Did you see anything there? \\
\hline & & & $22 \mathrm{~s}$ & I don't know... I started feeling something... I don't know, it's probably just me... \\
\hline & 2 & 2 & No & $\begin{array}{l}\text { Anything there? } \\
\text { No. }\end{array}$ \\
\hline & 3 & 2 & DTIp4 & Anything here? \\
\hline & & & $8 \mathrm{~s}^{\prime}$ & No. \\
\hline \multirow[t]{5}{*}{ DTlp2-1 } & 2 & 2 & No & Anything here? Do you feel anything, see anything? \\
\hline & & & & $\begin{array}{l}\text { Yeah, I feel like I... (looks perplexed, puts hand to forehead) I feel like I saw, like, some other site, we were at the train station.. } \\
\text { So it feels like you were in a subway? } \\
\text { Yeah, outside the train station. }\end{array}$ \\
\hline & 2 & 2 & No & Let me know if you get any sensation like that again. Do you feel anything here? No? \\
\hline & & & & No, I... (doesn't continue) \\
\hline & & & & $\begin{array}{l}\text { Did you see the train station, or did you feel like you were in the train station? } \\
\text { I saw it. }\end{array}$ \\
\hline \multirow[t]{11}{*}{ DTIp3-2 } & 2 & 2 & No & Let me know if you feel or see anything here. \\
\hline & & & & Again, I saw almost like a... I don't know, like I saw... It was very brief... \\
\hline & 2 & 2 & No & $\begin{array}{l}\text { I'm going to show it to you one more time. See if you can describe it any further. } \\
\text { Um... }\end{array}$ \\
\hline & 2 & 4 & No & I'm going to give it to you one last time. What do you think? \\
\hline & & & & $\begin{array}{l}\text { Um... I don't really know what to make out of it, but I saw like, another staircase... (pause) The rest I couldn't make out, but I } \\
\text { saw a closet space, but not this (points to the closet facing him in the hospital room), that one was stuffed, and it was blue. } \\
\text { Have you seen it before at some point in your life, you think? } \\
\text { Yeah. I mean, when I saw the train station... } \\
\text { The train station you'd been at. } \\
\text { Yeah. }\end{array}$ \\
\hline & 2 & 2 & No & Did you see it again? \\
\hline & & & & Yeah, I saw it again. \\
\hline & & & & Same thing, staircase and...? \\
\hline & & & & Yeah. \\
\hline & 4 & 2 & DTlp3-1 & It is disturbing, or is it just weird? \\
\hline & & & $8 \mathrm{~s}$ & It's kind of weird. \\
\hline \multirow[t]{7}{*}{ DTlp6-5 } & 4 & 2 & No & Let me know if you see, feel or hear anything here. \\
\hline & & & & I felt something like a little, almost like if there was a rubber band (points to his own face, shows his cheeks). \\
\hline & 4 & 2 & No & I'm going to do it for you again. \\
\hline & & & & (looks surprised, puts hand to forehead) Your... face looked differently at that moment. \\
\hline & & & & The faces looked different? Did they twist on you? \\
\hline & & & & Yeah, your faces were all... slightly different. \\
\hline & 3 & 2 & No & $\begin{array}{l}\text { (patient is instructed to look straight at the experimenter's face) Let me know if it happens again. } \\
\text { I... (doesn't continue) }\end{array}$ \\
\hline \multirow[t]{9}{*}{ DTIp5-4 } & 3 & 2 & DTlp4 & Let me know if the same sort of thing happens again. \\
\hline & & & $14 \mathrm{~s}$ & It did again. \\
\hline & & & & (during the afterdischarge) Is it still happening? \\
\hline & & & & Um, no. Only for that brief moment. \\
\hline & & & & What about the rest of my body? \\
\hline & & & & $\begin{array}{l}\text { Yeah, I saw like... something else... (chuckles) I'm sorry... You all looked Italian... Like you were working in a pizza shop. } \\
\text { That's what I saw, aprons and whatnot. Yeah, almost like you were working in a pizzeria. (chuckles) }\end{array}$ \\
\hline & 2 & 2 & No & $\begin{array}{l}\text { Let me know if we're back in the pizzeria. } \\
\text { (smiles, but says) No, not again. }\end{array}$ \\
\hline & 3 & 2 & DTlp4 & Let me know if you see it here again. \\
\hline & & & $6 s$ & (chuckles) It was just a small change, but that's it, we went back. \\
\hline \multirow[t]{4}{*}{ DTIp7-6 } & 3 & 2 & No & Let me know if there is anything funny here. \\
\hline & & & & No. \\
\hline & 4 & 2 & No & Here? \\
\hline & & & & Nothing. \\
\hline \multirow[t]{4}{*}{ DTlp8-7 } & 4 & 2 & DTlp8-7 & Let me know if there's anything weird here. \\
\hline & & & $11 \mathrm{~s}$ & No. \\
\hline & 2 & 2 & DHb7 & Anything here? \\
\hline & & & $7 \mathrm{~s}$ & No. \\
\hline \multirow[t]{4}{*}{ DHb6-5 } & 2 & 2 & No & Anything there? \\
\hline & & & & A little bit of déjà vu. \\
\hline & 3 & 2 & $\mathrm{DHb} 5-4$ & I saw... Um, there was a strong déjà vu to it. It's a specific street around where I live. It's called (name of the street). \\
\hline & & & & $\begin{array}{l}\text { Did you see the street, or did it just feel like you were there? } \\
\text { I saw it. }\end{array}$ \\
\hline
\end{tabular}


Table 1. Continued

\begin{tabular}{|c|c|c|c|c|}
\hline Site & $\begin{array}{l}\text { Amplitude } \\
(\mathrm{mA})\end{array}$ & $\begin{array}{l}\text { Duration } \\
\text { (s) }\end{array}$ & ADs? & Unedited transcripts of the patient's and experimenters' dialogue \\
\hline \multirow[t]{2}{*}{ DHb4-3 } & 4 & 2 & DHb4-3 & Anything here? \\
\hline & & & $5 s$ & $\begin{array}{l}\text { Yeah, I'm having that again. I saw that place and I felt the déjà vu... And I remember what I was thinking about. I think I was } \\
\text { seeing... It was like my eye doctor's office or something like that. (later says it is an optometrist's office) }\end{array}$ \\
\hline \multirow[t]{2}{*}{ DHb2-1 } & 1 & 2 & No & $\begin{array}{l}\text { Let me know if anything happens here. } \\
\text { A little bit of déjà vu. }\end{array}$ \\
\hline & 2 & 2 & $\begin{array}{l}\text { DHb2-1, } \\
\text { DHh2-1 } \\
4 \mathrm{~s}\end{array}$ & (no further dialogue regarding this site) \\
\hline
\end{tabular}

For each electrode pair, the stimulation amplitude and duration are tabulated, along with the location and duration of any afterdischarges (ADs) and the dialogue between the patient and the experimenters. The stimulation sites are presented in chronological order. Between stimulation of DTIp8-7 and DHb6-5, several other sites (not reported here) were stimulated (see Materials and Methods).

DTIp1 were located inside the fMRI-defined area, with DTIp2-1 closest to the peak activation. Face-selective visual responses were more posterior and lateral in both hemispheres with left predominance (data not shown) and were not sampled by any electrodes.

The location of the intracranial electrodes is shown in Figure $1 A$. During the iEEG experiment, the patient correctly detected $72 \%$ of the images that occurred twice in a row. He never responded to nontarget images. The median (IQR) response time was 594 (124) ms. iEEG analysis revealed that electrode pairs DTIp2-1 and DTIp3-2 were clearly house-selective, as demonstrated by a sustained, significantly larger BBGP increase in response to houses than to other categories (Fig. $1 C, D$ ). DHb4-3 also displayed selectivity for houses. One electrode pair (DTIp5-4) showed a significant early selective BBGP increase to faces and a later, brief, selective response to places.

\section{Direct electrical stimulation of house-selective visual areas elicits topographic visual hallucinations}

The locations and effects of electrical stimulation are summarized in Figure $1 B-D$ and detailed in Table 1 . Stimulation of DTIp2-1, in the medial fusiform gyrus, an area that was houseselective according to both fMRI and iEEG, caused the patient to experience a complex, topographic visual hallucination: he reported seeing a train station in the neighborhood where he lives. When questioned, the patient reported seeing the scene as if it was in front of him, not having the feeling of being there. Stimulation of DTIp3-2, more laterally in the fusiform gyrus, elicited another topographic visual hallucination with different contents: the patient reported seeing a scene that included a staircase and a stuffed, blue-colored closet space. That closet was different from the one facing his bed in his hospital room. It was unclear whether the staircase and closet were part of a coherent visual scene or a mixture of two different scenes, and if these objects were familiar to the patient.

Stimulation of DTIp6-5, in the inferior temporal gyrus, caused the patient to perceive the faces of the investigators present in his room as distorted, as if their features were twisted. There was no other change in the patient's visual perception. DTIp6-5 did not display face-selective iEEG visual responses. However, stimulating DTIp5-4, which showed iEEG early face and later place selectivity, evoked a complex visual phenomenon that included both face-body components and a topographic context: the patient reported that the investigators present in the room suddenly "all looked Italian, like you were working in a pizza shop, with aprons and whatnot." Afterdischarges involving DTIp4 were present at the stimulation amplitude used $(3 \mathrm{~mA})$, but the percept only persisted during the actual stimulation.

Stimulating DHb4-3 and DHb6-5 generated a strong sensation of déjà vu, identical with the patient's usual auras, together with a topographic visual hallucination of a street scene in the neighborhood where the patient lives, including an optometrist's office. At both sites, afterdischarges involving DHb4 were observed. Stimulating DHb2-1 induced déjà vu without any visual percept. The patient also experienced déjà vu without any visual percept upon stimulation of the amygdala and entorhinal cortex (data not shown).

Thirty-eight additional electrode pairs in the temporal and frontal lobes of the right hemisphere were stimulated (97 trials) at similar amplitudes and for similar durations. In no instance did the patient report any visual percept.

\section{Irritative and seizure onset zone}

Video-iEEG monitoring demonstrated five seizures with onset from the right amygdala and hippocampus. Frequent interictal spike and wave discharges were seen from the right anterior medial temporal lobe (amygdala, hippocampus, parahippocampal gyrus) and right anterior lateral temporal neocortex. None of the electrodes in the DTIp shaft were part of the irritative or seizure onset zones. The patient underwent a tailored right temporal lobectomy. Nine months after surgery, he has only experienced auras.

\section{Discussion}

\section{Consequences of direct electrical stimulation of place- selective cortical areas}

To our knowledge, this is the first time that topographic visual hallucinations were elicited by direct electrical stimulation of a functionally identified place-selective cortical area. Our results provide causal evidence that neural activity in the PPA, and the anatomical and functional neuronal network that is likely also recruited by stimulation (Selimbeyoglu and Parvizi, 2010), subtends the visual perception of places. The fact that specific places were seen suggests that the PPA is directly involved in the perception of visual scenes, rather than dealing exclusively with contextual or higher-order representations thereof.

The abundance of afterdischarges in this patient prevented us from extensively assessing the reliability of stimulation effects or from detailing the patient's percepts by testing, for instance, what would happen if we had stimulated the PPA while he was looking at faces. This is especially relevant if we consider that the patient reported the same percept upon repeat stimulation of some sites ("staircase + closet," DTIp3-2), but not others ("train station," DTIp2-1). This suggests that the effects of PPA stimulation may be dependent on the patient's cognitive state, train of thought, or sensory input. Further research will be needed to elucidate this point.

On one occasion, stimulation of two distinct sites (DHb6-5 and DHb4-3) gave rise to an identical percept ("optometrist's 
office + déjà vu"). In both cases, afterdischarges were found to involve DHb4, which might have been the true locus of the percept.

We did not perform sham stimulation trials. However, the fact that the patient did not report any visual percept upon 97 stimulations of 38 electrode pairs outside DTIp and DHb reinforces the specificity of the patient's subjective reports.

The patient's peculiar report of "pizza shop workers" (DTIp5-4) occurred on stimulating the lateral aspect of the fMRI-defined PPA, a site that displayed early iEEG selectivity to faces and later to houses. Interestingly, objects that are strongly associated with a precise topographic context activate the PPA even in the absence of any background (Bar and Aminoff, 2003). It is tempting to speculate that the stimulated area integrated visual information about faces or people and places. That stimulating immediately laterally (DTIp6-5) caused distortion of face perception is compatible with this hypothesis, but there are important caveats: the effect was not reproducible, and the stimulated area did not correspond to the fMRI and iEEG-defined fusiform face area, whose stimulation was shown by Parvizi et al. (2012) to distort face perception. In any case, it will be interesting to investigate the functional role of the cortex at the boundary of place- and face-selective areas.

Murphey et al. (2009) previously reported that stimulating a place-selective electrode in one patient generated an elementary visual percept ("a little explosion"), while stimulating an electrode that responded to faces as well as places in another patient failed to elicit any percept. Several factors may explain these discrepant findings. First, we stimulated a place-selective cortical area in the right hemisphere. Virtually all patients with acquired topographic disorientation have right hemispheric lesions (Landis et al., 1986; Epstein et al., 2001). Further, the probability of experiencing visual phenomena upon electrical stimulation of depth electrodes is higher in the right hemisphere than in the left (Jonas et al., 2013).

Second, the overall probability of eliciting a complex visual percept upon direct electrical stimulation of temporal cortex is low (Penfield and Perot, 1963). Further, the probability of perceiving any visual phenomena decreases as the stimulated visual cortical area lies further away from the occipital pole (Murphey et al., 2009; Jonas et al., 2013). Third, the physiological effects of electrical brain stimulation remain poorly understood, and it is recognized that stimulating what looks like the same site in different patients may yield different results (Borchers et al., 2011; Desmurget et al., 2013). Indeed, microstimulation experiments in animals have demonstrated that slight differences in the position of the stimulating electrode can lead to large differences in the pattern of responsive neurons (Histed et al., 2009). Thus, differing behavioral responses to stimulation of macroscopically similar sites could be due to small but significant variations in electrode position. Fourth, we used stereo-EEG electrodes, which are completely embedded into brain tissue and can contact cortex in sulcal banks and fundi, whereas subdural electrodes only contact gyral crowns. This is relevant in light of recent findings that scene-selective cortex may center on the collateral sulcus rather than the crown of the parahippocampal gyrus (Nasr et al., 2011). Depth electrodes can contact white as well as gray matter, which could reduce spatial specificity through stimulation of white matter tracts. Here, all electrodes were in contact with cortex or in its immediate vicinity, and the effects of stimulation corresponded well to the functional mapping, arguing against such a loss of spatial specificity.

\section{Neuronal networks underlying topographic visual hallucinations}

Topographic visual hallucinations can be triggered by stimulation of several cerebral structures. Penfield and Perot (1963) reported visual hallucinations, akin to memories, that included scenes during intraoperative cortical stimulation of the temporal lobe, mostly on the hemispheric convexity of the right hemisphere. Gloor et al. (1982) and Vignal et al. (2007) elicited complex visual hallucinations upon stimulation of the amygdala and hippocampus in patients implanted with stereo-EEG electrodes, without the implication of the lateral temporal neocortex. Bartolomei et al. (2004) stressed the role of entorhinal and perirhinal cortex in déjà vu and reminiscences of scenes from memory. By contrast, Bancaud et al. (1994) noted that stimulation of either the medial temporal lobe or the temporal neocortex could trigger a "dreamy state" (which could include both complex visual hallucinations and déjà vu), with afterdischarges spreading from the stimulated site (whether medial or lateral) to its counterpart in most cases. They thus suggested that the dreamy state was subtended by activity in a network that included medial and lateral temporal lobe. The network even extends beyond that, as suggested by two patients in whom stimulation of the left prefrontal cortex elicited visual hallucinations including scenes and people (Blanke et al., 2000).

The above-mentioned work also suggests that the distinction between complex visual hallucinations and déjà vu is not straightforward. Our patient differentiated the déjà vu (identical to his auras) from the visual hallucinations that he experienced during electrical stimulation (which were never part of his seizures). We thus suggest that déjà vu and visual hallucinations are two distinct phenomena that may occasionally occur together and that could be subtended by partly distinct, partly overlapping neuronal networks.

\section{Direct electrical stimulation as a tool in cognitive neuroscience}

The usefulness of direct electrical stimulation to probe human cerebral function has recently been questioned (Borchers et al., 2011). Our stimulation results converged with both fMRI and iEEG. We believe that such multimodal concordance contributes to reaffirm the merits of direct electrical cerebral stimulation as a unique tool in the armamentarium of the cognitive neuroscientist (Parvizi et al., 2012; Desmurget et al., 2013).

\section{References}

Aguirre GK, Zarahn E, D’Esposito M (1998) An area within human ventral cortex sensitive to "building" stimuli: evidence and implications. Neuron 21:373-383. CrossRef Medline

Bancaud J, Brunet-Bourgin F, Chauvel P, Halgren E (1994) Anatomical origin of deja vu and vivid "memories" in human temporal lobe epilepsy. Brain 117:71-90. CrossRef Medline

Bar M, Aminoff E (2003) Cortical analysis of visual context. Neuron 38: 347-358. CrossRef Medline

Bartolomei F, Barbeau E, Gavaret M, Guye M, McGonigal A, Régis J, Chauvel P (2004) Cortical stimulation study of the role of rhinal cortex in deja vu and reminiscence of memories. Neurology 63:858-864. CrossRef Medline

Bastin J, Committeri G, Kahane P, Galati G, Minotti L, Lachaux JP, Berthoz A (2013a) Timing of posterior parahippocampal gyrus activity reveals multiple scene processing stages. Hum Brain Mapp 34:1357-1370. CrossRef Medline

Bastin J, Vidal JR, Bouvier S, Perrone-Bertolotti M, Bénis D, Kahane P, David O, Lachaux JP, Epstein RA (2013b) Temporal components in the parahippocampal place area revealed by human intracerebral recordings. J Neurosci 33:10123-10131. CrossRef Medline

Benjamini Y, Hochberg Y (1995) Controlling the false discovery rate: a 
practical and powerful approach to multiple testing. J R Stat Soc Ser B 57:289-300.

Blanke O, Landis T, Seeck M (2000) Electrical cortical stimulation of the human prefrontal cortex evokes complex visual hallucinations. Epilepsy Behav 1:356-361. CrossRef Medline

Bokil H, Andrews P, Kulkarni JE, Mehta S, Mitra PP (2010) Chronux: a platform for analyzing neural signals. J Neurosci Methods 192:146-151. CrossRef Medline

Borchers S, Himmelbach M, Logothetis N, Karnath HO (2011) Direct electrical stimulation of human cortex- the gold standard for mapping brain functions? Nat Rev Neurosci 13:63-70. CrossRef Medline

Davidesco I, Zion-Golumbic E, Bickel S, Harel M, Groppe DM, Keller CJ, Schevon CA, McKhann GM, Goodman RR, Goelman G, Schroeder CE, Mehta AD, Malach R (2013) Exemplar selectivity reflects perceptual similarities in the human fusiform cortex. Cereb Cortex, in press. CrossRef Medline

Desmurget M, Song Z, Mottolese C, Sirigu A (2013) Re-establishing the merits of electrical brain stimulation. Trends Cogn Sci 17:442-449. CrossRef Medline

Epstein RA (2008) Parahippocampal and retrosplenial contributions to human spatial navigation. Trends Cogn Sci 12:388-396. CrossRef Medline

Epstein R, Kanwisher N (1998) A cortical representation of the local visual environment. Nature 392:598-601. CrossRef Medline

Epstein R, Deyoe EA, Press DZ, Rosen AC, Kanwisher N (2001) Neuropsychological evidence for a topographical learning mechanism in parahippocampal cortex. Cogn Neuropsychol 18:481-508. CrossRef Medline

Gloor P, Olivier A, Quesney LF, Andermann F, Horowitz S (1982) The role of the limbic system in experiential phenomena of temporal lobe epilepsy. Ann Neurol 12:129-144. CrossRef Medline

Groppe DM, Urbach TP, Kutas M (2011) Mass univariate analysis of eventrelated brain potentials/fields I: a critical tutorial review. Psychophysiology 48:1711-1725. CrossRef Medline

Histed MH, Bonin V, Reid RC (2009) Direct activation of sparse, distributed populations of cortical neurons by electrical microstimulation. Neuron 63:508-522. CrossRef Medline

Jenkinson M, Smith S (2001) A global optimisation method for robust af- fine registration of brain images. Med Image Anal 5:143-156. CrossRef Medline

Jenkinson M, Beckmann CF, Behrens TE, Woolrich MW, Smith SM (2012) FSL. Neuroimage 62:782-790. CrossRef Medline

Jonas J, Frismand S, Vignal J-P, Colnat-Coulbois S, Koessler L, Vespignani H, Rossion B, Maillard L (2013) Right hemispheric dominance of visual phenomena evoked by intracerebral stimulation of the human visual cortex. Hum Brain Mapp 10.1002/hbm.22407:n/a-n/a.

Landis T, Cummings JL, Benson DF, Palmer EP (1986) Loss of topographic familiarity: an environmental agnosia. Arch Neurol 43:132-136. CrossRef Medline

Murphey DK, Maunsell JH, Beauchamp MS, Yoshor D (2009) Perceiving electrical stimulation of identified human visual areas. Proc Natl Acad Sci U S A 106:5389-5393. CrossRef Medline

Nasr S, Liu N, Devaney KJ, Yue X, Rajimehr R, Ungerleider LG, Tootell RB (2011) Scene-selective cortical regions in human and nonhuman primates. J Neurosci 31:13771-13785. CrossRef Medline

Papademetris X, Jackowski M, Rajeevan N, Okuda H, Constable RT, Staib LH (2011) BioImage Suite: an integrated medical image analysis suite, Section of Bioimaging Sciences, Dept. of Diagnostic Radiology, Yale School of Medicine. http://www.bioimagesuite.org.

Parvizi J, Jacques C, Foster BL, Withoft N, Rangarajan V, Weiner KS, GrillSpector K (2012) Electrical stimulation of human fusiform faceselective regions distorts face perception. J Neurosci 32:14915-14920. CrossRef Medline

Penfield W, Perot P (1963) The brain's record of auditory and visual experience: a final summary and discussion. Brain 86:595-696. CrossRef Medline

Selimbeyoglu A, Parvizi J (2010) Electrical stimulation of the human brain: perceptual and behavioral phenomena reported in the old and new literature. Front Hum Neurosci 4:46. CrossRef Medline

Smith SM (2002) Fast robust automated brain extraction. Hum Brain Mapp 17:143-155. CrossRef Medline

Vignal JP, Maillard L, McGonigal A, Chauvel P (2007) The dreamy state: hallucinations of autobiographic memory evoked by temporal lobe stimulations and seizures. Brain 130:88-99. CrossRef Medline 